\title{
A Japanese Text Input Interface Using On-Line Writing-Box-Free Handwriting Recognition and Kana-to-Kanji Conversion
}

\author{
Takeshi Sakurada, Yoichi Hagiwara, Hideto Oda, and Masaki Nakagawa \\ Tokyo University of Agriculture and Technology, \\ 2-24-16, Naka-cho, Koganei-shi, Tokyo, Japan \\ take-s@cc.tuat.ac.jp
}

\begin{abstract}
People often forget how to write Kanji characters even though they are able to read those characters. If one cannot write a Kanji character, one cannot input it with current handwriting recognition engines. Therefore, we propose to combine Kana (phonetic characters) to Kanji conversion with a handwriting recognition engine. Moreover, we remove writing boxes to write characters one by one, since they are unnatural for many applications. This is similar to writing on a plain paper with a pen. When, handwritten characters are misrecognized, the user corrects them using correction gestures. When the user cannot write Kanji characters, the user writes in Kana and invokes the Kana-toKanji conversion. In reality, people often write Kana characters instead of Kanji characters when they cannot write those characters or it takes more time than writing Kana characters. Kana-to-Kanji conversation is common in keyboard interface. This paper describes the design of a pen interface by which the user can write text without writing boxes, correct misrecognitions and specify Kanato Kanji conversion. When a user writes Kana characters instead of Kanji characters, it would be desirable to translate them to Kanji characters automatically.
\end{abstract}

Keywords: pen interface, free-format handwriting recognition, Kana-to-Kanji conversation.

\section{Introduction}

Pen-based interfaces are attracting people's attention again due to the wide spread of mobile PDAs (Personal Digital Assistant) with pen input, expanding sales of interactive electronic whiteboards, shipping of Tablet PC of Microsoft Corp., etc. Especially, Nintendo DS, which is a portable game machine with pen, is opening a new market in Japan.

Pen input has several benefits. It does not require large space. People can use it without training. Pen input on interactive whiteboards can easily attract the attention of an audience. People can express or annotate their ideas without being bothered to wonder how to use a pen. Thinking is not interrupted by the actions for writing. However, there are still some problems with existing handwritten character recognition. 
One problem is that handwriting recognition engines might make misrecognitions. Another problem is that many systems require people to write characters one by one into so-called writing boxes in order to avoid segmentation errors. However, a user likes as few restrictions as possible, when writing. There is also another problem. People often forget how to write Kanji characters even though they are able to read those characters. It takes time to write complex Kanji characters. If one cannot write a Kanji character, one cannot input it with current handwriting recognition engines.

Therefore, we propose to combine Kana (phonetic characters) to Kanji conversion with a handwriting recognition engine. Moreover, we remove writing boxes to write characters one by one, since they are unnatural for many applications. This is similar to writing on a plain paper with a pen.

\section{Lazy Recognition and Correction by Gestures}

We have been working on on-line writing-box-free handwriting recognition [1][2]. A user can write text without using writing boxes, as if writing on a plain paper. Even with the latest character recognition engine, however, the correct recognition rate is $93 \%$ and incorrect recognitions occur and the correction work is surely needed. Some of systems have adopted the method of recognizing and showing a recognition result immediately after a character is written [3]. This uses the real-time nature of on-line input. However, it forces a user to verify a recognition result and correct misrecognition when necessary, thus interrupts user's thinking. Therefore, we have proposed the lazy recognition [4], which delay the display of recognition results of handwritten character patterns until needed.

In handwriting recognition, misrecognition is unavoidable. Therefore, recognition error correction interface is necessary. Error correction by pen gestures is effective. We have already proposed a set of pen gestures for writing-box-free handwriting input [5][6].

This section summarizes the state of the art and our previous studies.

\subsection{A Character Recognition with Writing-Boxes}

The character recognition with writing-boxes is a system, which is generally used by the present PDA etc. and recognizes by filling in one character at a time to one frame (Fig.1). The recognized result may be mistaken, although character segmentations become right since the character recognition with a frame recognizes a character for every frame. Therefore, what is necessary is to consider only the interface, which corrects the mistake of the recognized result. For example, the candidate of a recognition result is taken out with carrying out the tap of the inside of the frame which wrote in the character with an electronic pen, and a user is made to choose the right result from there. Since the character recognition with writing-boxes has the right character segmentations, it can raise the rate of recognition. However, the burden that a character must be written to a frame is given to a user. 


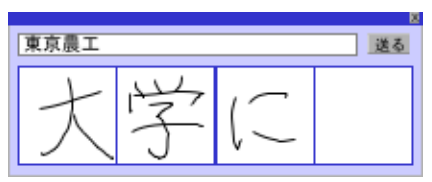

Fig. 1. Character recognition with writing-boxes

\subsection{A Writing-Box-Free Handwriting Recognition}

Writing-box-free character recognition recognizes that which the user took notes of freely as a character string, and there is no input frame in every character (Fig.2).

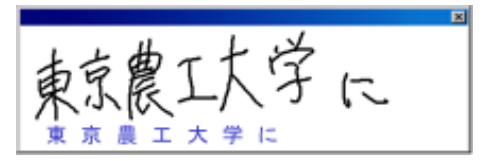

Fig. 2. A writing-box-free handwriting recognition

Writing-box-free character recognition may incorrect-recognize a character simple substance like recognition with a writing-box. Furthermore, since writing-box-free character recognition does not have a frame for every character, character segmentations may be incorrect-recognized. When character segmentations have been incorrect-recognized, though natural, it becomes what also mistook the last result (Fig.3).

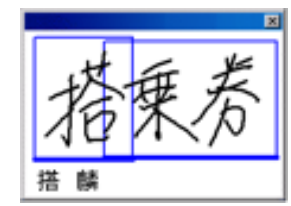

Fig. 3. Incorrect-recognized character segmentations

\subsection{The Display of the Recognition Result of Character Segmentations}

As mentioned above, in writing-box-free character recognition, there is a problem that character segmentations are sometimes incorrect-recognize. Therefore, in order to correct character segmentations, it is necessary to indicate how the character recognition system has recognized character segmentations to a user. The following two methods are mentioned as a method of displaying character segmentations.

1. The color of a stroke is changed and segmentations are displayed (Fig.4).

2. A rectangle (frame) is displayed and segmentations are displayed (Fig.5). 


\section{京都钼光案内}

Fig. 4. Character segmentations are expressed with the color of strokes

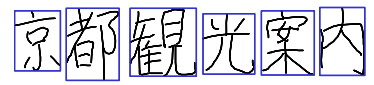

Fig. 5. Character segmentations are expressed using a rectangle

In this paper, the method of displaying character segmentations using a rectangle is adopted. It is because there may be a case of monochrome environment by PDA etc. or a color may be used by emphasis of a stroke etc. also in a color environment.

\subsection{Correction of Character Segmentations}

Since character segmentations were accidentally detected by writing-box-free character recognition, the incorrect-recognized example is shown in Fig.6 and Fig.7. The example shown in Fig.6 is an example, which has incorrect-recognized the pattern which recognition engine should recognize as two characters as one character. In this case, a user performs the gesture, which draws a line and divides a rectangle in a place to divide a pattern (Fig.6-b). Then, a character is divided in the corrected place and a new recognition result is displayed (Fig.6-c). By the gesture that divides this rectangle, both the starting point and the ending point of a division line are a rectangular outside. Moreover, when the place, which should be recognized as one character, has been divided like the example of Fig.7-a, the gesture surrounding a stroke to set to one is carried out (Fig.7-b). Then, a character recognition system corrects the boundary of a character, and displays a new recognition result (Fig.7-c).
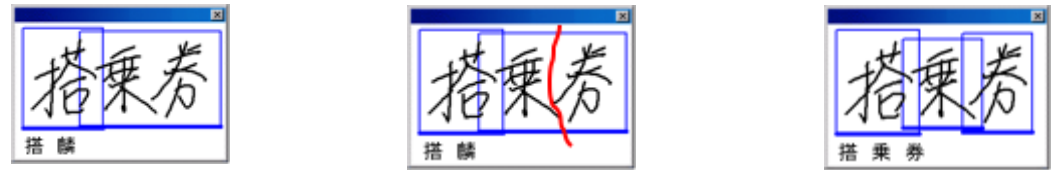

(a)Segmentations incorrect (b)Correction of segmentations (c)New recognition result

Fig. 6. Incorrect recognition of character segmentations (it is intension about excessive strokes)
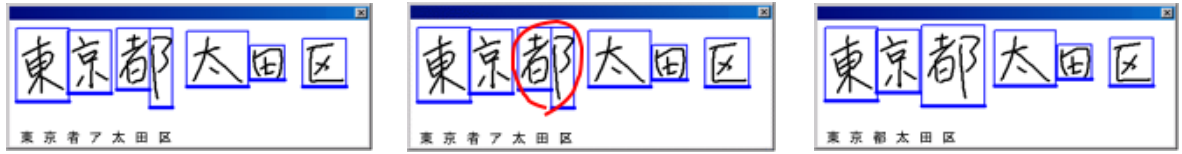

(a)Segmentations incorrect (b)Correction of segmentations (c)New recognition result

Fig. 7. Incorrect recognition of character segmentations (when dividing too much) 


\subsubsection{The Evaluation Experiment of the Correction Gesture of Character Segmentations}

In order to confirm the validity of the above-mentioned character segmentations correction gesture, we experimented in preliminary evaluation. Although connection gesture (Fig.8) was used in former study [7] in order to have made the divided character into one character, we experimented this time by adding the gesture that was defined above in addition to it.

The evaluation experiment was conducted on ten university students who are skilled in computer operation and pen operation using Tablet PC. Before beginning an evaluation experiment, explanation of a display interface and a correction interface is given, and we operated the system to them freely and had them get used.

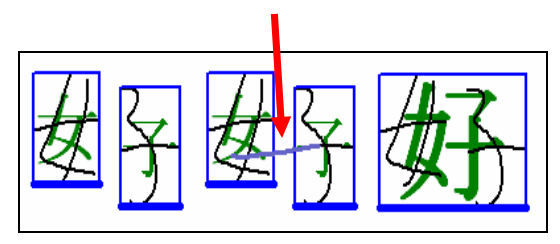

Fig. 8. Combination of the character by connection gesture (former system)

In the input of about 20 characters, both the gesture to divide and the gesture to surround were used about 1.5 averages as a result of the experiment. However, connection gesture was not used once. Since the gesture to surround is more nearly intuitive than connection gesture, it is thought that the gesture to surround was used. From this experiment, it supposes that connection gesture will not be adopted from now on, and this gesture is used for the below-mentioned conversion-of-kana-intokanji input gesture.

\section{Conversion-of-Kana-into-Kanji Input Gesture}

If the above-mentioned user interface is used, while a user corrects incorrect recognition, it will become easy to input a character into a system. However, the character inputted into the system is not necessarily a character that the user wanted to input truly. As a result of our cooperating in the experiment for program work of NHK (Japan Broadcasting Corporation) before, it recognizes that the character, which can be read and which can be written are not the same. In the input of Japanese by the keyboard, the conversion of kana into kanji is performed and the kanji character is inputted. In this case, if reading is known even when memory of a character is ambiguous, reading is inputted and the target kanji character can be inputted by the conversion of kana into kanji. However, since in handwriting input it cannot write if the kanji character is not known in detail even if reading is known, a user cannot input a character into a system. Since the writing order is sharply different or a line and a point are lacking, the kanji character currently regarded as the ability of a user to 
write may not be exact in fact, either. Therefore, in character recognition, the kanji character, which the user desired, is not displayed as a recognition result. Therefore, a user inputs kana characters unavoidably instead of a character to input first. In order to solve this situation, the interface for using the conversion of kana into kanji in a handwriting input is designed.

\subsection{Combined Use of Incorrect Recognition Correction and Character Segmentations Correction}

It is possible to change the mode like character segmentation correction mode, incorrect recognition correction mode, conversion-of-kana-into-kanji mode, and then to use conversion-of-kana-into-kanji input gesture at the time of conversion-of-kanainto-kanji mode. In this case, a user always needs to grasp in which mode it is working now, and has the time-consuming problem of a mode change.

In this paper, the conversion-of-kana-into-kanji input gesture, which can perform correction of character segmentations and revision of incorrect recognition, is proposed, without changing the mode.

\subsection{An Addition of a Conversion-of-Kana-into-Kanji Candidate}

Before describing conversion-of-kana-into-kanji input gesture, the incorrect recognition correction gesture used by the conventional character recognition with a frame [5][6] is described. In the conventional incorrect recognition correction, when the user did the tap of the inside of the rectangle to which the recognized character was written as shown in Fig. 9, the recognition candidate list was displayed and the user had chosen the right thing out of it (Fig.9-d).
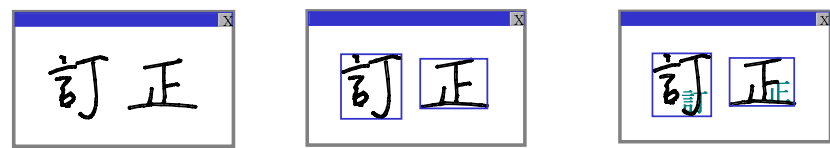

(a)Stroke input (b)Character segmentation (c) recognition result

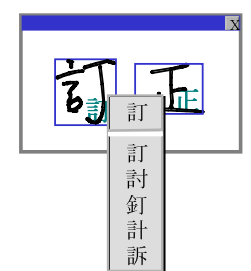

(d)Correction of a recognition result

Fig. 9. The conventional incorrect recognition correction

By this system, it extends by adding a conversion-of-kana-into-kanji result to this conventional incorrect recognition correction gesture. First, the example in one character is shown to Fig.10. The stroke input which the user inputted is recognized (Fig.10-b,c). Usually, character segmentation recognition result and character recognition result are displayed almost simultaneous. If a user does the tap of the inside of the rectangle to which the recognized character was written, the candidate list of recognition results and a conversion-of-kana-into-kanji result will be 


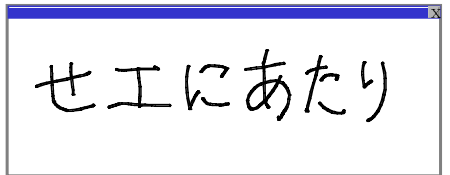

(a) Stroke input

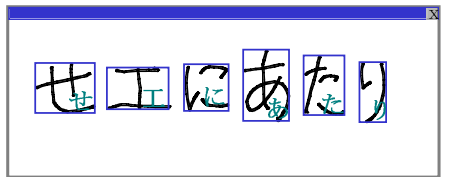

(c) Character recognition result

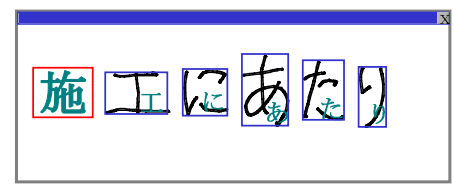

(e) Conversion-of-kana-into-kanji result

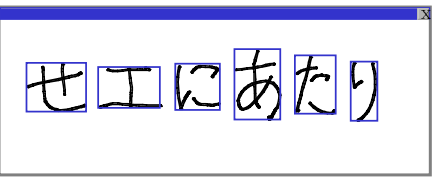

(b) Character segmentation recognition result

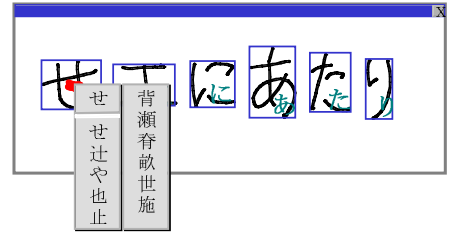

(d) Recognition result correction and the conversion of kana into kanji

Fig. 10. The addition of the conversion of kana into kanji (one character)

displayed, and the thing which a user wants to input out of them will be chosen (Fig.10-d,e).

\subsection{The Addition of the Gesture for the Conversion of Kana into Kanji}

Although the above-mentioned thing converts one kana character to a kanji character, actually a conversion-of-kana-into-kanji system converts it to kanji characters from two or more kana characters in many cases. Therefore, it must be able to use together with the gesture for choosing two or more characters, and the gesture of correction of character segmentations, and correction of a character recognition result.

When both the starting point and the ending point of gesture are contained in some rectangle, the stroke in the rectangle which gesture passed is set as the object of the conversion of kana into kanji. An example is shown in Fig.11. First, the stroke (Fig.11-a), which the user inputted, is recognized (Fig.11-b,c). Usually, the recognition result of character segmentations and character recognitions result are displayed continuously. If a user does the tap of the inside of the rectangle surrounding the recognized character, correction of a character recognition result and selection of a simple sentence character conversion-of-kana-into-kanji result are possible. Correction of the above-mentioned character segmentations can be performed by the gesture, which surrounds strokes or divides a rectangle at this time. When the starting point and the ending point of gesture are contained in the respectively different rectangle (Fig.11-d), the portion which gesture passed is converted to kanji characters from the kana characters (Fig.11-e). Finally, the result of the conversion of kana into kanji is chosen and displayed (Fig.11-f). However, since it surrounds for correcting character segmentations when the starting point and the ending point of gesture are in the same rectangle, correction of the character segmentations instead of the conversion of kana into kanji is performed. 


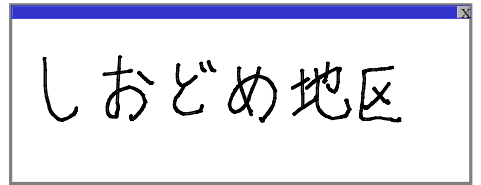

(a) Stroke input

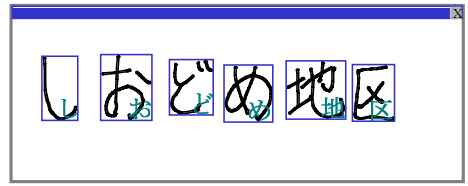

(c) Character recognition result

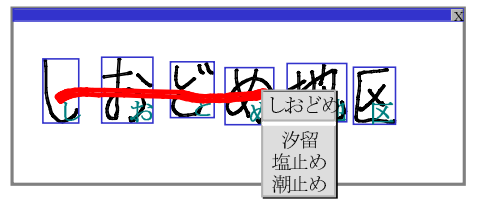

(e) Recognition result correction and the conversion of kana into kanji

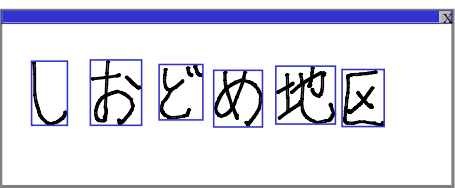

(b) Character segmentations recognition result

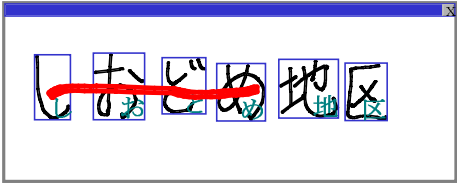

(d) Correction of a character boundary, conversion-of-kana-into-kanji position specification

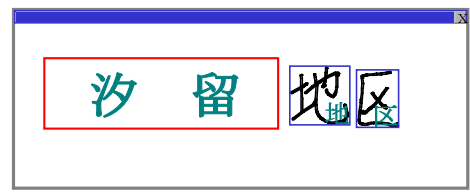

(f) Conversion-of-kana-into-kanji result

Fig. 11. The addition of the conversion of kana into kanji (Two or more characters)

The flowchart of the interface, which added the gesture for the conversion of kana into kanji, is shown in Fig.12.

A user applies recognition processing to arbitrary timing to the inputted stroke to write. Recognition engine presumes character segmentations first, performs character recognition, and displays the character string of a result. If a user inputs the character segmentations correction gesture, character segmentations will be presumed again and a character recognition result will be displayed. When character recognition results differ, character recognition result correction gesture, which carries out the tap of the circumscription rectangle of a character, is performed. Then, below the next candidate of character recognition result and the result of the simple sentence character conversion of kana into kanji are displayed. A user chooses from what was displayed and performs correction of a character recognition result, and the simple sentence character conversion of kana into kanji. When a user inputs the gesture, which performs the conversion of kana into kanji, a conversion-of-kana-into-kanji candidate is displayed from two or more selected Kana characters, and a user chooses. Character recognition and a conversion-ofkana-into-kanji result are decided by the above-mentioned procedure.

By the method of proposing in this paper, character recognition and the conversion of kana into kanji can be performed to a user's arbitrary timing. For this reason, the following becomes possible. When it is in a hurry, or when there is no necessity of carrying out character recognition, it saves with the handwritten stroke. When there is time, or when it is necessary to recognize and to make it a printing type, character recognition can be carried out using the saved stroke, and a result can be used. Since 


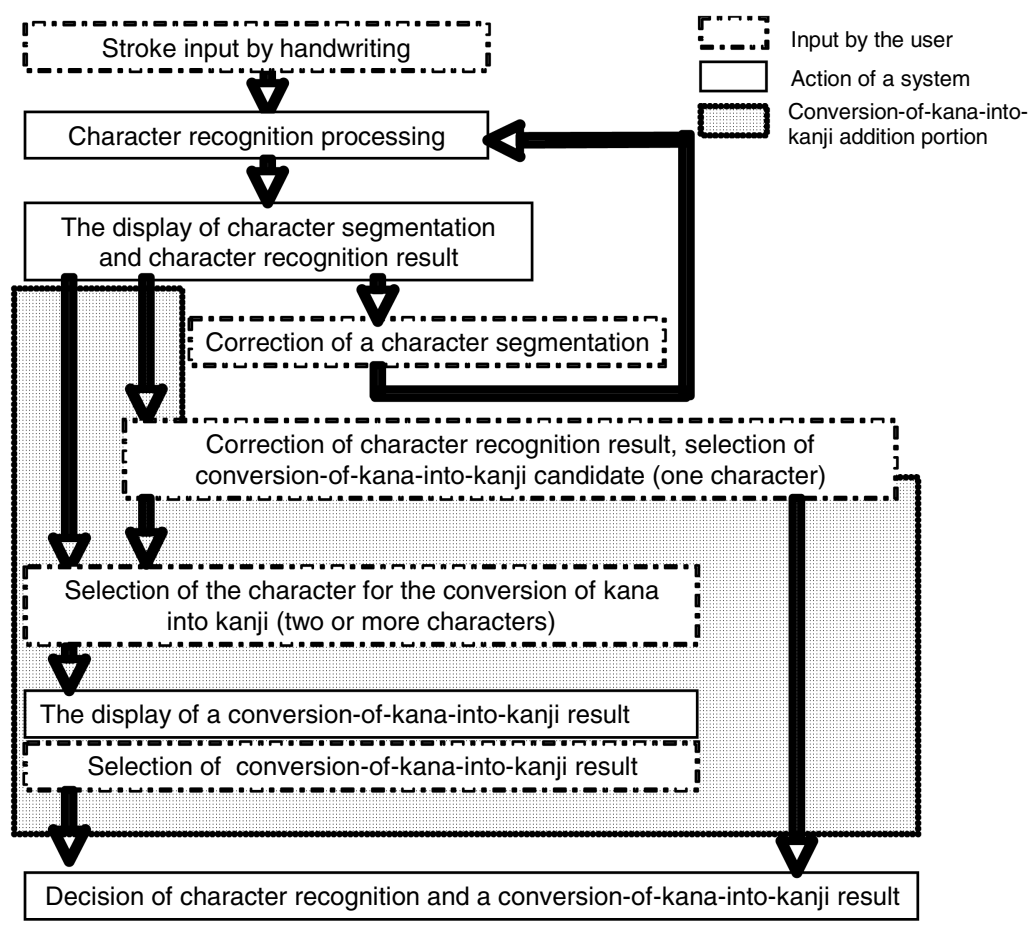

Fig. 12. This systems flowchart

it can carry out by the gesture which neither correction of recognition nor the conversion of kana into kanji overlaps, a user does not need to change mode and needs to perform neither correction nor conversion.

\section{Extendibility}

As extension of this design, improvement using a context analysis in recognition accuracy and improvement using a prediction input in input speed can be considered. The system independent of client environment can be built by processing character recognition and the conversion of kana into kanji by the server installed on the network. Furthermore, collection of a handwriting character pattern and collection of the character string, which carried out the conversion of kana into kanji, become possible on a server. Although we have already started for construction of a character recognition server [8], it is necessary to realize in the form incorporating a conversion-of-kana-into-kanji server from now on.

\section{Conclusion}

This paper described the design of the input interface using on-line writing-box-free handwriting recognition and Kana-to-Kanji conversion. During a handwriting input, if 
this interface is used, when a kanji character is not known, it writes in kana characters and the conversion of kana into kanji can be carried out later. A free input is attained without barring thinking in frame-less handwriting input environment by using the interface described in this paper.

\section{References}

1. Fukushima, T., Nakagawa, M.: On-line Writing Box Free Recognition of Handwritten Text based on Stochastic Models. IEICE Technical Report, PRMU 98-139(489), 25-30 (1998)

2. Oki, T., Nakagawa, M.: Implementation and Evaluation of a Combined Recognition System using Normalization of Likelihood Values. Technical Report for IEICE Japan, PRMU2002196 102(555), 43-48 (2003)

3. Fukushima, T., Yamada, H.: A Predictive Pen-Based Japanese Text Input Method and Its Evaluation. Trans. of IPS Japan 37(1), 23-30 (1996)

4. Souya, T., Fukushima, H., Takahashi, N., Nakagawa, M.: A Basic Design of Handwriting User Interface with Lazy Recognition. Trans. of IPS Japan 34(1), 158-166 (1993)

5. Bandoh, H., Fukushima, T., Kato, N., Nakagawa, M.: User interfaces for correcting errors in writing-box-free recognition of handwritten text. IPSJ SIG Notes, 2000-HI-89, pp. 81-88 (2000)

6. Bandoh, H., Fukushima, T., Kato, N., Nakagawa, M.: User interfaces for correcting errors in writing-box-free recognition of handwritten text. Trans. of IPS Japan 43(6), 1996-2005 (2002)

7. Nakagawa, M., Yorifuji, M.: Error Correction Gestures for Free-format Text Input by Pen Interfaces. In: Proc. Human Interface Symposium 2005, vol. 1, pp. $43-46$ (2005)

8. Sakurada, T., Yorifuji, M., Onuma, M., Nakagawa, M.: Web-based Applications Using PenBased Interfaces and Network-Based on-line Handwriting Recognition. In: HumanComputer Interaction 10th International Conference (HCI International 2003), vol. 2, pp. 268-272 (2003) 